\title{
Sodium Alginate Mediated Route for the Synthesis of Monodisperse Silver Nanoparticles Using Glucose as Reducing Agents
}

\author{
Dong Chunfa, ${ }^{1,2}$ Zhang Xianglin ${ }^{1}, \quad$ Cai Hao ${ }^{1}, \quad$ Cao Chuanliang ${ }^{1}$ \\ ${ }^{1}$ State Key Laboratory of Materials Processing and Die \& Mould Technology, Huazhong University of Science and Technology, Wuhan 430074, \\ China; ${ }^{2}$ Hubei Polytechnic University, Huangshi 435003, China
}

\begin{abstract}
A simple and environmentally friendly method was developed for preparing colloidal silver nanoparticles in aqueous solutions using silver nitrate, sodium alginate and glucose as silver precursor, capping agents and reducing agents, respectively. The formation of silver nanoparticles was observed by the change of color from colorless to yellow. The silver nanoparticles were characterized by transmission electron microscopy (TEM), UV-visible spectroscopy (UV-vis) and X-ray diffraction (XRD). The TEM images show that the particles are small, well dispersed and spherical in shape with a narrow distribution from $3 \mathrm{~nm}$ to $12 \mathrm{~nm}$. The XRD demonstrates that the obtained metallic nanoparticles are single crystalline silver nanoparticles. The effects of the reaction time, the reaction temperature and the concentration of silver ion and reducing agent on the particle size were investigated by the UV-vis spectra. The present process is an excellent candidate for the preparation of silver nanoparticles because it is simple, free-pollutant, inexpensive and easy to perform. The method may be extended to other noble metals, such as $\mathrm{Au}, \mathrm{Pd}$ and $\mathrm{Pt}$, for medicinal, industrial and technological applications.
\end{abstract}

Key words: silver nanoparticles; sodium alginate; green synthesis

Metal nanoparticles have attracted much attention over the past decades because of their special physical and chemical properties and potential applications ${ }^{[1-6]}$. Among metal nanoparticles, silver nanoparticles have aroused widespread interest as they have wide areas of applications such as catalyst ${ }^{[7]}$, antimicrobial agents ${ }^{[8]}$, conductive coating and sensors ${ }^{[9,10]}$. Many interesting methods have been developed to synthesis of nanoparticles, such as chemical reduction $^{[11]}$, sonochemical reduction ${ }^{[12]}$, photo reduction $^{[13]}$, microwave processing ${ }^{[14]}$, and laser ablation ${ }^{[15]}$. Among these methods, chemical reduction method is the most extensively used because of its simplicity, low cost, and the ease of size and shape control over silver nanoparticles. Sodium borohydride ${ }^{[16]}$, hydrazine hydrate ${ }^{[17]}$, aniline $^{[18]}$ and dimethylformamide ${ }^{[19]}$ are frequently used as reducing agents for the synthesis of silver nanoparticles. However, these reducing agents may cause significant environmental pollution. Thus, there is a growing need to develop a environmentally friendly method for synthesis of silver nanoparticles, the process of which would not involve the toxic chemicals. A green method employing either microorganisms or nature source has emerged as a simple and cost-effective environmentally friendly viable alternative to chemical synthetic procedures ${ }^{[8,20-22]}$. The use of nature source for the synthesis of silver nanoparticles could be more advantageous due to the absence of biohazards and the culture of the microorganisms. Silver nanoparticles have been synthesized using various nature source such as polysaccharide ${ }^{[23]}$, maltose ${ }^{[24]}$, chitosan ${ }^{[25]}$, arabic gum ${ }^{[26]}$, source ${ }^{[27]}$ and plant extracts ${ }^{[4,28]}$. Khan et al.,

$\overline{\text { Received date: February 25, }} 2015$

Foundation item: 7th European Community Framework Marie Curie International Research Staff Exchange Scheme (IRSES) Program (FP7-PEOPLE-2010-IRSES, 269113); China-European Union Technology Cooperation (1110, (2011))

Corresponding author: Zhang Xianglin, Ph. D., Professor, School of Materials Science and Engineering, Huazhong University of Science and Technology, Wuhan 430074, P. R. China, Tel: 0086-27-87558148,E-mail: hust_zxl@mail.hust.edu.cn 
reported green synthesis of silver nanoparticles using starch as capping agents due to its good biocompatibility and biodegradability $^{[29]}$. Venkatpurwar et al., reported green synthesis of silver nanoparticles using sulfated polysaccharide as stabilizer ${ }^{[23]}$. They used sodium hydroxide and hydrochloric acid as accelerator, which may be associated with environmental toxicities or biological hazards. Oluwafemi et al., reported green synthesis of silver nanoparticles using gelatin as stabilizer, and maltose as reducing agent, with no accelerator being used. But $42 \mathrm{~h}^{\text {[24] }}$ had been taken in the synthesis process, which is considered to be a long time. Hence, a new cleaner and easier feasible technique for synthesizing silver nanoparticles is desired

Taking advantage of these literatures into account, the main purpose of the present study is to produce a new green simple method to synthesis of monodisperse silver nanoparticles with narrow distribution by environmentally friendly materials. Sodium alginate (NaAlg), a type of natural polymer with excellent biocompatibility and biodegradability, is composed of mannuronic acid (M block) and guluronic acid (G block) units. Glucose, is a renewable, inexpensive and nontoxic reducing agent, comprising aldehyde group which reduces the $\mathrm{Ag}^{+}$to $\mathrm{Ag}^{0}$ in the preparation of silver nanoparticles. It is worth emphasizing that silver nanoparticles stabilized by sodium alginate using glucose as reducing agent can be easily integrated into systems relevant for biomedical and pharmaceutical applications.

In the present work, we reported a totally green synthesis of uniform and stable silver nanoparticles via a single-step reduction of silver ions using glucose, a nontoxic sugar as the reducing agent and sodium alginate as stabilizer, and nothing as accelerator in order to take advantages of properties of components and use them in biomedical and pharmaceutical applications. Compared to the gelatin stabilized silver nanoparticles, the sodium alginate stabilized silver nanoparticles can be obtained in less time. Moreover, the synthesis is very simple, inexpensive and eco-friendly benign, devoid of complex processes such as irradiation, photochemical, or electrochemical processes. To the best of our knowledge, it is the first time here to use sodium alginate as stabilizer, glucose as the reducing agent, for synthesis of silver nanoparticles.

\section{Experiment}

Silver nitrate $(99.9 \%$, analytical grade) purchased from Sinopharm Chemical Reagent Co., China, was used as the precursor of the formation of silver nanoparticles. Sodium alginate (analytical grade) purchased from Aladdin, Shanghai Jingchun Reagent Co., China, was used as the capping agent. Glucose (analytical grade) purchased from Shanghai Chemical Reagent Co., China, was used as reducing agent. All chemicals were used as received without further purification and doubly distilled water was used in all experiments. All glasswares used in the laboratory experiments were cleaned with a fresh solution of $\mathrm{HNO}_{3} / \mathrm{HCl}$ (3:1, volume ratio), washed thoroughly with doubly distilled water, and dried before use.

For all experiments, the source of silver was silver nitrate in distilled water. The preparation of silver nanoparticles was simply achieved by reduction of silver nitrate with glucose in aqueous solution. In a typical experiment, $1 \mathrm{~g}$ sodium alginate was added to $50 \mathrm{~mL}$ distilled water in a flask and the solution was stirred and heated to $50{ }^{\circ} \mathrm{C}$ to obtain a clear solution. $10 \mathrm{~mL}$ AgNO3 solution (0.005 mol/L) was added to the sodium alginate solution with continuous stirring to obtain $\mathrm{Ag}+/ \mathrm{NaAlg}$ solution. This was followed by the addition of $40 \mathrm{~mL}$ glucose solution ( $5 \mathrm{wt} \%$ ) with continuous stirring (keeping in mind that the total volume of the reaction medium was $100 \mathrm{~mL}$ ). The solution was maintained at $80{ }^{\circ} \mathrm{C}$ and allowed to react for $10 \mathrm{~h}$. In order to monitor the growth of the silver nanoparticles, aliquots were taken at different time intervals. For investigation of the role of glucose and temperature in particles size of silver nanoparticles, the experiment was also carried out at different concentrations of glucose and different temperatures.

The synthesized colloidal silver nanoparticles were characterized with the help of a UV-vis spectrophotometer (Cary 50 scan). The UV-vis absorption spectra were recorded at room temperature by placing the sample in a 1 $\mathrm{cm}$ quartz cuvette over wavelength of $200 \mathrm{~nm}$ to $800 \mathrm{~nm}$ operating at a resolution of $1 \mathrm{~nm}$. The size and the morphology of the silver nanoparticles were characterized by JEM 2100 transmission electron microscope (TEM) operating at an acceleration voltage of $200 \mathrm{kV}$. TEM samples were prepared by placing a drop of diluted colloidal silver nanoparticles solution onto copper grids covered by carbon film, and the solvent was evaporated at room temperature. X-ray powder diffraction (XRD) measurements were carried out on X'Pert PPO X-ray diffractometer with $\mathrm{CuK} \alpha$ radiation of wavelength of $\lambda=0.15406 \mathrm{~nm}$ in the $2 \theta$ range of $10^{\circ}$ to $90^{\circ}$ with a scanning rate of $0.05 \% \mathrm{~s}$ at room temperature.

\section{Results and Discussion}

\subsection{Effects of time and temperature of reduction on silver nanoparticles}

As many types of colloidal nanomaterials, silver nanoparticles also require organic ligands in the preparation to avoid nonreversible aggregation in solution. It is known that sodium alginate solution is a suitable capping agent of noble metal nanoparticles due to its good biocompatibility and biodegradability. Hence, we used sodium alginate to synthesize and stabilize silver nanoparticles. Besides stabilizing the nanoparticles and preventing them from 
aggregation, sodium alginate makes the silver nanoparticles dispersible in aqueous solution. The colour of the $\mathrm{Ag}^{+} / \mathrm{NaAlg}$ solution was slowly changed from transparent to yellowish brown as the reaction time goes on, which is related to the formation of silver nanoparticles. The colour of the samples prepared by sodium alginate is enhanced with the increase of reaction time, the concentration of $\mathrm{AgNO}_{3}$, the temperature and the concentration of glucose.

The gradual evolution of silver nanoparticles was investigated by UV-vis spectroscopy. Fig.1A displays the UV-vis absorption spectra taken at different reaction time intervals of continuous transformation of silver ions into the sodium alginate capped silver nanoparticles in the typical procedure. After $0.5 \mathrm{~h}$ of reaction, a pale yellow solution, which displayed a broad surface plasmon resonance (SPR) peaked at $419 \mathrm{~nm}$ corresponding to the SPR of silver nanoparticles, can be observed, confirming the formation of silver nanoparticles with broad size distribution (Fig.1A-b). The solution turns to dark yellow gradually with the accumulation of reaction time from $0.5 \mathrm{~h}$ to $7 \mathrm{~h}$, which increases the corresponding peak intensities with a concomitance of small blue shifts from $419 \mathrm{~nm}$ to $411 \mathrm{~nm}$ (Fig.1A-c $\sim$ g, Fig.1B). The increase in intensities suggests the increase in the concentration of the silver nanoparticles and continued reduction of silver ions. The small blue-shift and the decrease of the broadness of the SPR peak indicate the decrease in the size with a narrow size distribution. This phenomenon can be explained as following: firstly, as the silver ions are reduced to silver atoms, many silver nuclei are formed. The newly formed silver nuclei will precipitate to silver nanoparticles with certain size due to the presence of capping agents. And the SPR peak is found at $419 \mathrm{~nm}$. As the time goes on, more and more silver nuclei are formed and relatively smaller silver nanoparticles are generated, and the average size is reduced. Hence, there is a blue-shift as the reaction time goes on. After $7 \mathrm{~h}$ of reaction, the peak and its intensity reach and remain the same (Fig.1A-h, Fig.1B), indicating the reduction reaction comes to an end.

To study the effects of temperature on silver nanoparticles formation, samples with the same concentrations of silver ion and glucose were prepared and heated for $7 \mathrm{~h}$ at different temperatures. Fig.2A and Fig.2B shows the effects of temperature on extent of absorption of aqueous silver nanoparticles. It is clear that upon increasing temperature from $60{ }^{\circ} \mathrm{C}$ to $80{ }^{\circ} \mathrm{C}$, the absorption value of SPR is increased with small blue shift from $415 \mathrm{~nm}$ to 411 $\mathrm{nm}$. It is also observed that when the reaction temperature is blew $60{ }^{\circ} \mathrm{C}$, there is no absorption in visible region, indicating no formation of silver nanoparticles. The result suggests that the reaction temperature plays a vital role in synthesis of sodium alginate capped silver nanoparticles. At relatively higher temperature $\left(80{ }^{\circ} \mathrm{C}\right)$, at beginning, the


Fig.1 UV-vis spectra of silver nanoparticles as a function of reaction time (A); effect of reaction time on the absorbance of silver nanoparticles (B)
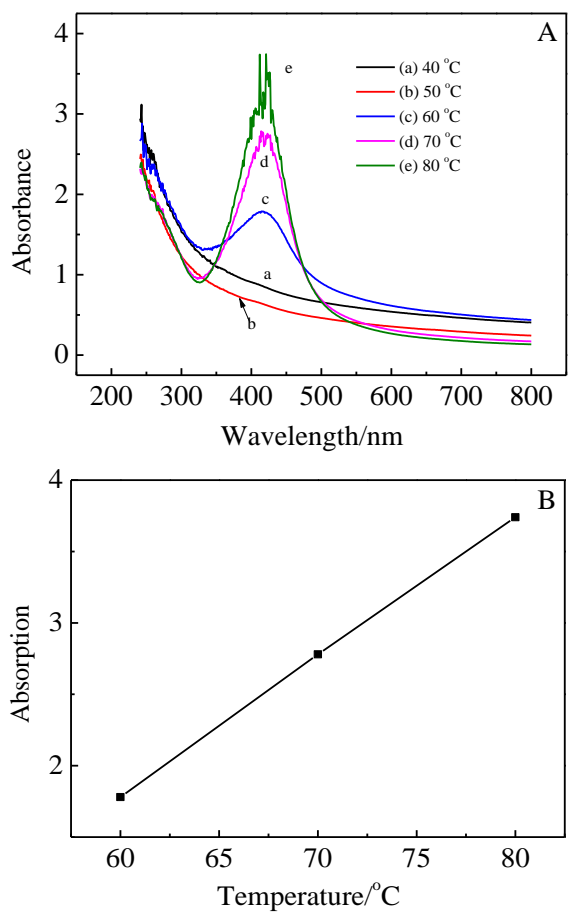

Fig.2 UV-vis spectra of silver nanoparticles as a function of reaction temperature $(\mathrm{A})$; effect of reaction temperature on the absorbance of silver nanoparticles (B)

number of silver nuclei is large enough to lower the concentration of silver ions, which lead to fast nucleation and slow growth. Under these conditions, the resulting silver nanoparticles are relatively monodisperse and small. 
However, when the reaction is performed at $60{ }^{\circ} \mathrm{C}$, it will lead to a slow nucleation and a slow growth, resulting in relatively bigger particles.

\subsection{Effects of glucose and silver nitrate concentrations}

In order to study the effect of glucose concentration on silver nanoparticles preparation, samples containing $0.5 \mathrm{wt} \%$ to $10 \mathrm{wt} \%$ of glucose were added into the $\mathrm{AgNO}_{3} / \mathrm{NaAlg}$ solution. The effect of glucose concentration is represented in Fig.3A and Fig.3B. It is clearly observed that silver colloidal obtained at the lowest glucose concentration exhibits yellow, showing a small SPR peak at $415 \mathrm{~nm}$ (Fig.3A-a). As the glucose concentration increases from $0.5 \mathrm{wt} \%$ to $5 \mathrm{wt} \%$, the color of the solution gradually changes to dark yellow and the SPR peak is small-blue-shifted from $415 \mathrm{~nm}$ to $411 \mathrm{~nm}$. Upon the increase in its intensity to a certain value, its broadness decrease. The increase of the peaks intensity implies that the concentration of silver nanoparticles increases. The symmetric and narrow absorption peak indicates the narrow size distribution of the silver nanoparticles at higher glucose concentration. As mentioned above, upon increasing of the glucose concentration, the reducing rate of silver ions is improved, the number of silver nuclei is large enough to lower the concentration silver ions, and hence, the resulting silver nanoparticles are small and monodisperse. However, when the glucose concentration changes from $5 \mathrm{wt} \%$ to $10 \mathrm{wt} \%$, the intensity of the SPR peak stays in the same certain value, in which a marginal change takes place.
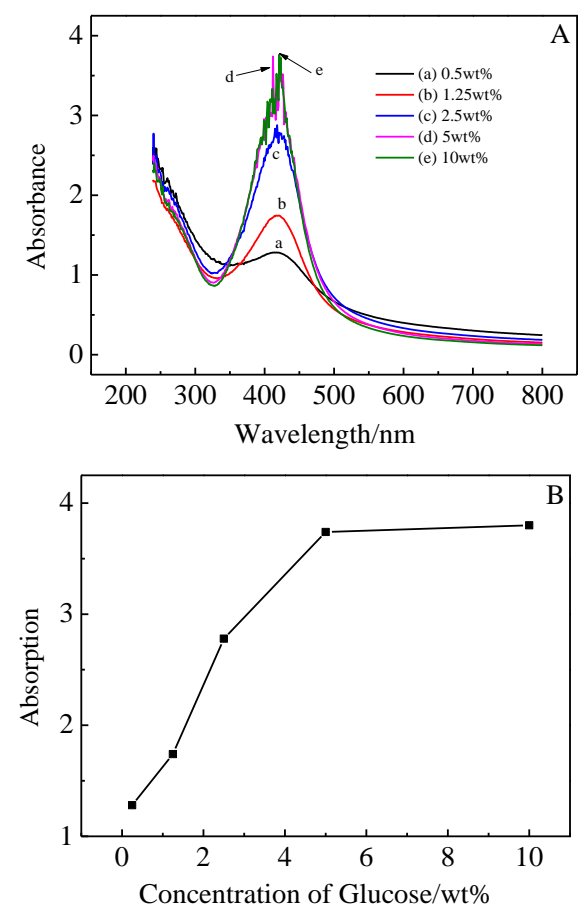

Fig.3 UV-vis spectra of silver nanoparticles as a function of glucose concentration (A); effect of glucose concentration on the absorbance of silver nanoparticles (B)
Silver nitrate as the precursor of silver nanoparticles is anticipated to have a ratable impact on the absorption value of the plasmon band. The effect of silver nitrate concentration on the evolution of silver nanoparticles in terms of its plasmon band intensity is shown in Fig.4A and Fig.4B. It is clear that as silver nitrate concentration increases from $1.25 \mathrm{mmol} / \mathrm{L}$ to $5 \mathrm{mmol} / \mathrm{L}$, the absorption value of SPR increases in a proportional manner. The increase of peaks intensity indicates the increase of concentration of silver nanoparticles. At relatively higher silver nitrate concentration, more silver nuclei are formed during the nucleation, which lead to the formation of more silver nanoparticles.

\subsection{TEM analysis}

TEM measurements were also conducted in order to estimate the particle size and the size distribution of the as-synthesized silver nanoparticles. The typical TEM images taken from drop coated films of the silver nanoparticles synthesized by treating silver nitrate solution with different amounts of sodium alginate are shown in Fig.5 and Fig.6. The TEM results demonstrate that the silver nanoparticles synthesized by higher amount of sodium alginate (1 g) are small, well dispersed and spherical in shape with a narrow size distribution from $3 \mathrm{~nm}$ to $12 \mathrm{~nm}$, and the silver nanoparticles synthesized by lower amount of sodium alginate $(0.2 \mathrm{~g})$ are irregular in shape with a broad size distribution. This is also confirmed by the UV-vis absorption spectra (Fig.7). With accumulation of the
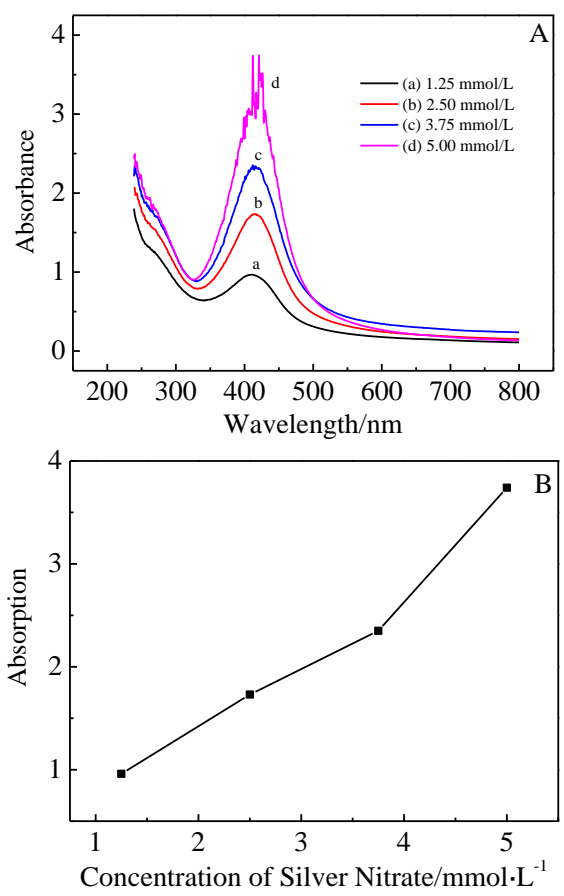

Fig.4 UV-vis spectra of silver nanoparticles as a function of silver nitrate concentration (A); Effect of silver nitrate concentration on the absorbance of silver nanoparticles (B) 


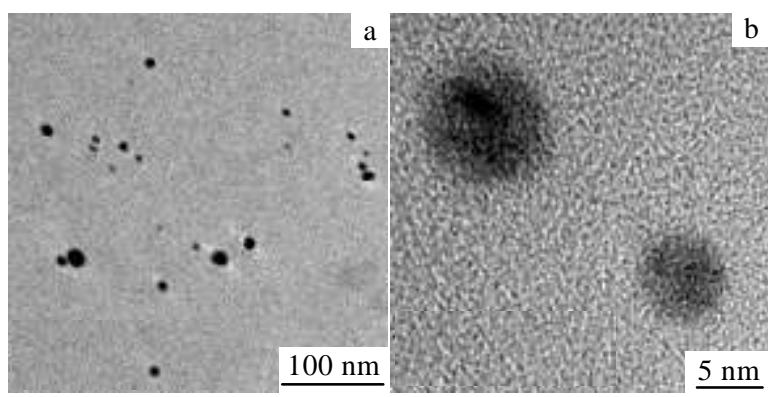

Fig.5 TEM image (a) and HRTEM image (b) of greensynthesized silver nanoparticles using sodium alginate $\left(\mathrm{AgNO}_{3}=5 \mathrm{mmol} / \mathrm{L}\right.$, glucose content $=5 \mathrm{wt} \%$, sodium alginate content $=1 \mathrm{~g}$, temperature $=80^{\circ} \mathrm{C}$, time $=7 \mathrm{~h}$ )

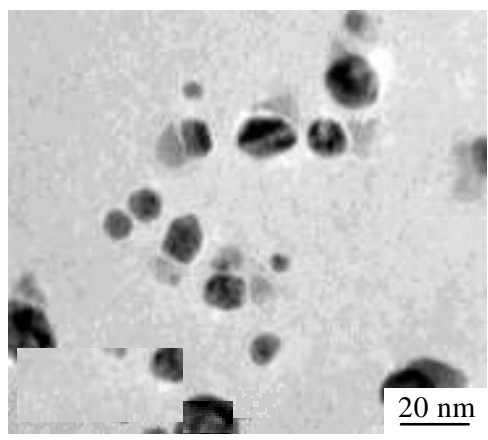

Fig.6 TEM image of green-synthesized silver nanoparticles using sodium alginate $\left(\mathrm{AgNO}_{3}=5 \mathrm{mmol} / \mathrm{L}\right.$, glucose content $=5 \mathrm{wt} \%$, sodium alginate content $=0.2 \mathrm{~g}$, temperature $=80^{\circ} \mathrm{C}$, time $=7 \mathrm{~h}$ )

amount of sodium alginate, the SPR peak is slightly blue shifted from $415 \mathrm{~nm}$ to $411 \mathrm{~nm}$ with increase in its intensity. The HRTEM image (Fig.5b) suggests that the as-prepared silver nanoparticles are spherical in shape and composed of well-ordered single crystals with distinct lattice fringes, which confirms that the spherical particles are highly crystalline.

\subsection{XRD patterns}

X-ray diffraction (XRD) has proven to be a useful research tool to testify the synthesis of silver nanoparticles and confirm the crystal structure of the as-synthesized silver nanoparticles. The typical XRD pattern of the as-prepared silver nanoparticles is shown in Fig.8, demonstrating the crystalline structure of the silver nanoparticles. A number of strong Bragg reflections can be seen at $38.24^{\circ}, 44.35^{\circ}, 64.68^{\circ}, 77.55^{\circ}$ and $81.66^{\circ}$, corresponding to the (111), (200), (220), (311) and (222) planes of the face-centered cubic crystalline structure of metallic of metallic silver, respectively (JCPDS No. 01-1167). No diffraction peaks corresponding to the precursor and/or bi-products are observed, confirming pure
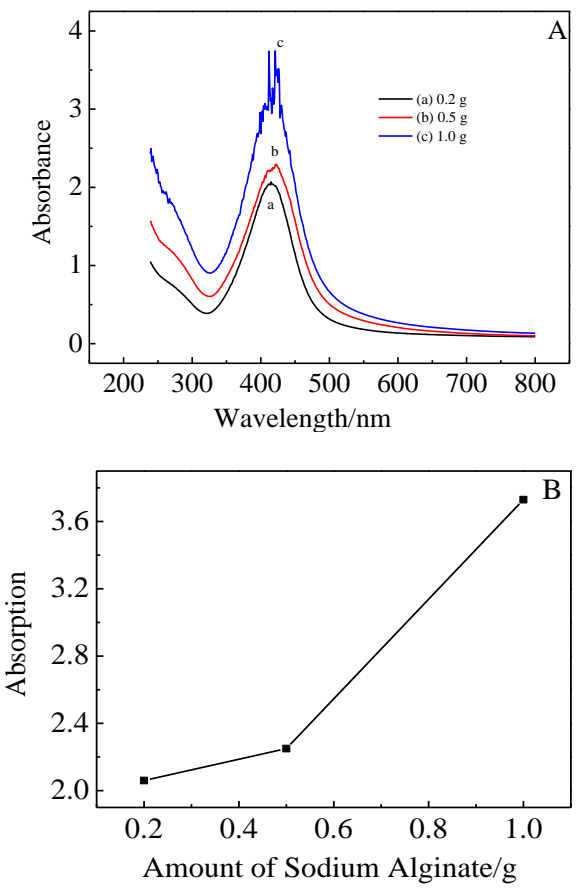

Fig.7 UV-vis spectra of silver nanoparticles as a function of amount of sodium alginate (A); effect of amount of sodium alginate on the absorbance of silver nanoparticles (B)

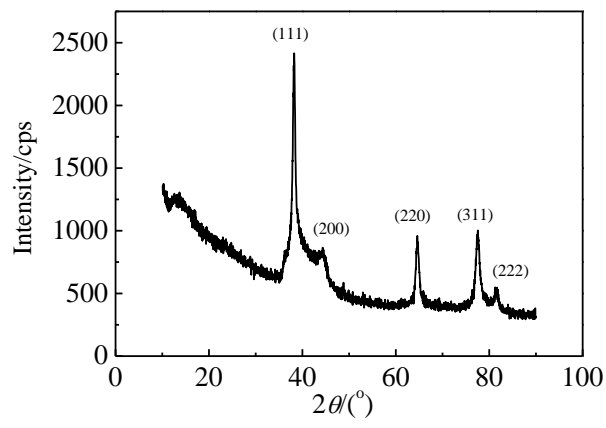

Fig.8 XRD pattern of silver nanoparticles

metallic silver is formed. The intensity of peaks reflects the high crystallinity of the silver nanoparticles. The diffraction peaks are broad displaying that silver particles with small sizes are obtained. The background noise is attributed to the sodium alginate absorbed on the surface of the as-prepared silver nanoparticles. Due to the absorption of sodium alginate, the as-prepared silver nanoparticles maintain good oxidation resistance.

\section{Conclusions}

1) The green method based on the silver-mirror can be provided for the synthesis of silver nanoparticles using sodium alginate as capping agent and glucose as reducing 
agent, which is regarded as an environmental friendly, simple and efficient route for preparation of metallic nanoparticles.

2) The as-synthesized silver nanoparticles are small, well-dispersed, and spherical in shape, with a narrow size distribution from $3 \mathrm{~nm}$ to $12 \mathrm{~nm}$.

3) Reaction temperature, reaction time, the concentration of silver source and reducing agent and the amount of capping agents play vital roles in size and yields of silver nanoparticles.

4) As the reaction time, reaction temperature, the concentration of reducing agent and the amount of capping agents increase, silver nanoparticles become smaller and their concentrations are enhanced.

\section{References}

1 Das R, Nath S S, Bhattacharjee R. Journal of Luminescence [J], 2011, 131: 2703

2 Dong C, Cai H, Zhang X et al. Physica E[J], 2014, 57: 12

3 Zaheer Z, Malik M A, Al-Nowaiser F M et al. Colloids and Surfaces $B[\mathrm{~J}], 2010,81: 587$

4 Dong C, Zhou K, Zhang X et al. Mater Lett[J], 2014, 120: 118

5 Eluri R, Paul B. Mater Lett[J], 2012, 76: 36

6 Dong C, Zhang X, Cai H. J Alloy Compd[J], 2014, 583: 267

7 Yang J, Pan J. Acta Materialia[J], 2012, 60: 4753

8 Zhang Y, Cheng X, Zhang Y et al. Colloids and Surfaces A[J], 2013, 423: 63

9 Natsuki J, Abe T. Journal of Colloid and Interface Science[J], 2011, 359: 19

10 Skim I, Lee Y, Lee K J et al. Materials Chemistry and Physics[J], 2008, 10: 316
11 Wang H, Qiao X, Chen J et al. Colloids and Surfaces $A[\mathrm{~J}]$, 2005, 256: 111

12 Byeon J H, Kim Y W. Ultraso Sonochem[J], 2012, 19: 209

13 Courrol L C, Silva F R O, Gomes L. Colloids and Surfaces $A[\mathrm{~J}], 2007,305: 54$

14 Khan A, El-Tohi A M, Alrokayan S et al. Colloids and Surfaces $A[\mathrm{~J}], 2011,377: 356$

15 Darroudi M, Ahmad M B, Zamiri R et al. J Alloy Compd[J], 2011, 509: 1301

16 Liu J, Li X, Zeng X. J Alloy Compd[J], 2011, 377: 356

17 Zhang D, Yang H, Physica B[J], 2013, 415: 44

18 Khan Z, Al-Thabaiti S A, Obaid A Y et al. Colloids and Surfaces $B[\mathrm{~J}], 2011,82: 513$

19 Rao C P K, Trivedi D C. Synthetic Metals[J], 2005, 155: 324

20 Zaheer Z, Malik A M, Al-Nowaiser F M et al. Colloids and Surfaces $B[\mathrm{~J}], 2010,81: 587$

21 Abdel-Halim E S, Al-Deyab S S. Carbohydrate Polymers[J], 2011, 86: 1615

22 Rani P U, Rajasekharreddy P. Colloids and Surface A[J], 2011, 389: 188

23 Venkatpurwar V, Pokharkar V. Mater Lett[J], 2011, 65: 999

24 Oluwafemi O S, Lucwaba Y, Gura A et al. Colloids and Surfaces $B[\mathrm{~J}], 2013,102: 718$

25 Twn Y, Chen Y, Shih C. Powder Technol[J], 2008, 185: 251

26 Juby K A, Dwivedi C, Kumar M et al. Carbohydrate Polymers[J], 2012, 89: 906

27 Filippo E, Serra A, Buccolieri A et al. Journal of NonCrystalline Solids[J], 2010, 356: 344

28 Karuppiah M, Rajmohan R. Mater Lette [J], 2013, 97: 141

29 Khan Z, Singh T, Hussain J I et al. Colloids and Surfaces B[J], 2013, 102: 578

\title{
海藻酸钠为保护剂葡萄糖为还原剂合成单分散银纳米粒子
}

\author{
董春法 ${ }^{1,2}$, 张祥林 ${ }^{1}$, 蔡 吴 $^{1}$, 曹传亮 ${ }^{1}$ \\ (1. 华中科技大学 材料成形与模具技术国家重点实验室, 湖北 武汉 430074)
}

(2. 湖北理工学院, 湖北 黄石 435003)

\begin{abstract}
摘 要: 报道了一种在水溶液中的简单绿色法制备纳米银溶胶。硝酸银, 海藻酸钠素, 葡萄糖分别作为银源, 保护剂和还原剂。纳米溶 胶的颜色从无色变到黄色, 表明生成了纳米银粒子。利用透射电子显微镜（TEM）, 紫外可见光谱（UV-vis）和 X 射线衍射（XRD）对 样品进行了分析。TEM 图像表明, 所得到的银纳米颗粒为粒径较小, 分散性较好的球状粒子, 且分布范围较窄, 从 $3 \mathrm{~nm}$ 到 $12 \mathrm{~nm}$ 。 XRD 结果表明所得到的纳米金属粒子为单晶纳米银颗粒。通过 UV 光谱对反应时间、反应温度、银离子浓度及还原剂的浓度对粒径的影响进 行了研究。由于该方法具有无污染、简单、廉价等优点，因此可以作为一种较好制备纳米银粒子的方法。该方法还可以扩展到其它贵金 属的技术应用, 例如药用, 工业应用等。
\end{abstract}

关键词: 纳米银粒子; 海藻酸钠; 绿色合成

作者简介: 董春法, 男, 1982 年生, 博士生, 华中科技大学材料学院, 湖北 武汉 430074, 电话: 027-87558148, E-mail: dongchunfa@ 126.com 